\title{
SHOTER NOTICES
}

Die Theorie der Gruppen von endlicher Ordnung, mit Anwendungen auf algebraische Zahlen und Gleichungen sowie auf die Kristallographie. By Andreas Speiser. Berlin, Julius Springer, 1923. viii +194 pp.

This book is volume 5 of the series entitled Die Grundlehren der mathematischen Wissenschaften in Einzeldarstellungen mit besonderer Berücksichtigung der Anwendungsgebiete, of which the first volume appeared in 1921. In accord with the plan of the series special attention is given to applications, but the greater part of the work is devoted to the development of the fundamental theorems relating to the theory of the groups of finite order. The beginner who may find the developments too brief is referred to the second volume of Weber's Algebra and to Netto's Gruppen und Substitutionentheorie for more extensive treatments.

From the title of the series it is clear that the aim is to deal with fundamental theories rather than to present details. Differences of opinion naturally exist as regards what should be regarded as most fundamental. Some might regard the theory of the $\phi$-subgroups, which does not appear in the present volume, as more fundamental than some of the theories which do appear. In fact, some readers may not agree with a statement found on page 97 to the effect that the representation of groups by means of substitutions is the most important domain of group theory. Most readers will probably agree, however, that the material of the present volume has been, on the whole, wisely selected for the purposes in view, which seem to have included an introduction to the theories due to Frobenius. From this standpoint the present volume is especially useful.

The following list of the fifteen chapter headings may serve to exhibit the nature of the material treated: The foundations, invariant subgroups and factor-groups, abelian groups, conjugate subgroups, Sylow groups and p-groups, groups of crystallography, permutation groups, automorphisms, monomial groups, representation of groups by linear homogeneous substitutions, group-characteristics, applications of the theory of groupcharacteristics, arithmetical researches on substitution groups, groups of a given degree, and theory of equations.

Comparatively few errors seem to have escaped the notice of the author. Among the few that might possibly trouble the beginner are the following: On page 66 it is stated, in theorem 66, that the subgroups composed separately of all the substitutions of a transitive permutation group of degree $n$ which omit a given letter constitute a system of $n$ conjugate subgroups of index $n$. This is true only when these subgroups are actually of degree $n-1$. On page 76 it is stated that the cyclic invariant subgroup of index 2 of a dihedral group gives rise to outer isomorphisms. This is evidently not the case when the order of this dihedral group is 6,8 , or 12 . The first of these groups has no outer isomorphisms at all.

G. A. MiLher 